\title{
Large Eddy Simulations: Wall Functions with Forcing given by Backscatter from a Scale-Similarity Model
}

\author{
Lars Davidson $^{1}$ \\ Division of Fluid Dynamics, Department of Applied Mechanics, Chalmers \\ University of Technology,SE-412 96 Göteborg, Sweden lada@chalmers.se, \\ www.tfd.chalmers.se $/ \sim$ lada
}

\section{Abstract}

Developing accurate wall functions for LES is a great challenge. This is indeed also the case also for RANS. It can be argued that it should be easier to develop accurate wall functions for LES than for RANS. In a well-resolved LES using wall functions, the task of the wall function is to give an instantaneous wall shear stress; this does probably not need to be exactly correct. Compare with inlet fluctuating boundary conditions: there are many (infinite) ways to impose instantaneous fluctuations that will yield accurate mean flow and Reynolds stresses.

The present work presents LES with wall functions. Forcing taken from back scatter from a scale-similarity model is used in the wall-adjacent cells. The object is to increase and enrich the resolved turbulence in the wall region. The new approach is applied for fully developed channel flow and 3D hill flow. For channel flow, it is indeed found that the results are improved using forcing. However, for 3D hill flow, it is found that the effect of forcing is negligible.

\section{Introduction}

The main hindrance for doing accurate Large Eddy Simulations (LES) of wall-bounded flows is the requirement of high grid resolution in the near-wall region. The grid needs to be fine not only in the wall-normal direction but also in the wall-parallel directions. Furthermore, the size of the grid cells scale with Reynolds number meaning that the problem becomes worse the higher the Reynolds number. One way to circumvent the requirement of fine grid in the near-wall region is to use wall functions. The use of wall functions allows us to reduce the number of cells. Needless to say, we put large - even unrealistic - requirements on wall functions: we model the entire inner wall region consisting of the viscous region, the buffer region and the inner logarithmic region with one single computational cell. In addition to saving computational cells, wall function grids entail another attractive feature, namely the 
fact that the aspect ratio of the wall-adjacent cells is much smaller than in hybrid LES-RANS and wall-modelled DES. For a structured grid, the ratio of the streamwise cell side to the wall-normal one can in hybrid LES-RANS and wall-modelled DES easily reach several hundred. Furthermore, high aspect ratios often deteriorate the convergence rate of the iterative solution procedure.

Figure 1 presents LES with wall functions of channel flow at four different Reynolds numbers. As can be seen, the velocity is overpredicted. The reason is that the total (i.e. modelled plus resolved) shear stress is too low; or, rather, that too a large velocity gradient is required to obtain the $y-1$ profile of the total shear stress dictated by the time-averaged streamwise momentum equation. To reduce the $U^{+}$velocity, either the modelled or the resolved shear stress has to be increased. One way to generate additional resolved turbulence is to use forcing. This has previously been used both in LES and hybrid LESRANS $[1,5,6,11,12,15]$.

The present work is the first study in which forcing is applied together with wall functions. The object is to increase the resolved turbulence in the wall region. This will be done by adding backscatter (forcing) from a scalesimilarity model in the wall-adjacent cells.

A dissipative scale-similarity subgrid model was recently proposed in which only the dissipative part of the subgrid stresses was added to the momentum equations [4]. This was achieved by adding the gradient of the scale-similarity stress only when its sign agreed with that of the corresponding viscous term. This idea was later used the other way around as forcing in hybrid LESRANS [3]: only the part of a subgrid stress term that corresponds to back scatter was added to the momentum equations. The forcing triggers resolved turbulence.

In the present work, the latter approach is used in connection with wall functions based on the instantaneous log-law. Forcing is applied in the cells adjacent to the walls to stimulate the generation of resolved turbulence. The new approach is evaluated for fully developed channel flow at $R e_{\tau}=4000$, 8000,16000 and 32000 , and for 3D hill flow.

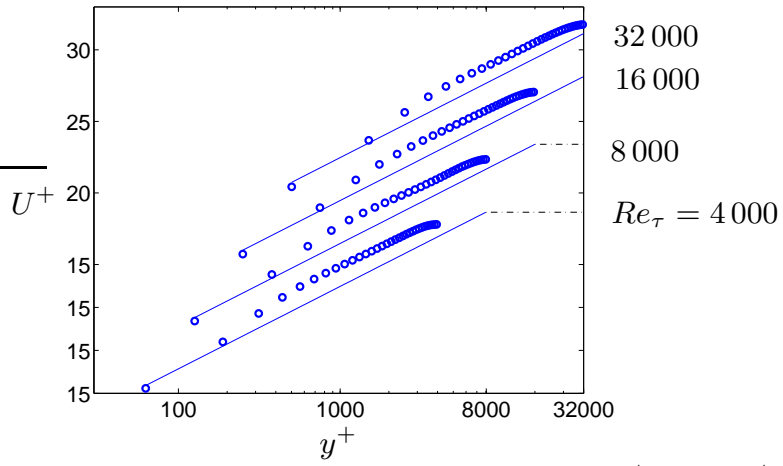

Fig. 1. LES using wall functions. Channel flow. ॰: LES; $\_: U^{+}=\left(\ln y^{+}\right) / 0.4+5.2$. 


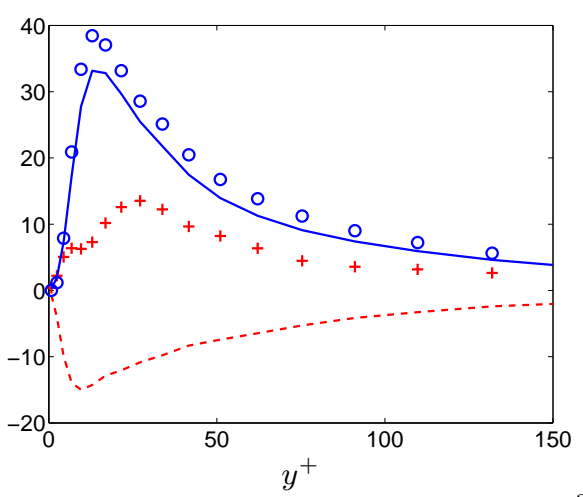

Fig. 2. Dissipation terms from DNS data taken from [5]. $96^{3}$ mesh data filtered onto a $48^{3}$ mesh. $R e_{\tau}=500$. $\_: \varepsilon_{S G S}^{+} ;---: \varepsilon_{S G S}^{-} ; \circ: \varepsilon_{S G S}^{D} ;+: \varepsilon_{S G S}$.

\section{Scale-similarity model}

\subsection{Dissipative model}

The filtered Navier-Stokes read (. denotes filtering)

$$
\frac{\partial \bar{u}_{i}}{\partial t}+\frac{\partial}{\partial x_{j}}\left(\bar{u}_{i} \bar{u}_{j}\right)+\frac{1}{\rho} \frac{\partial \bar{p}}{\partial x_{i}}=\nu \frac{\partial^{2} \bar{u}_{i}}{\partial x_{k} \partial x_{k}}-\frac{\partial \tau_{i k}}{\partial x_{k}}
$$

where $\tau_{i k}$ denotes the SGS stress tensor, which is modelled with the scalesimilarity model [14]

$$
\tau_{i k}=\overline{\bar{u}}_{i} \bar{u}_{k}-\overline{\bar{u}}_{i} \overline{\bar{u}}_{k}
$$

This model is not sufficiently dissipative. Let us take a closer look at the equation for the resolved, turbulent kinetic energy, $K=\left\langle u_{i}^{\prime} u_{i}^{\prime}\right\rangle / 2$, which reads $(\langle$.$\rangle denotes averaging in time)$

$$
\begin{gathered}
\frac{\partial K}{\partial t}+\frac{\partial}{\partial x_{j}}\left(\bar{u}_{j} K\right)+\left\langle u_{k}^{\prime} u_{i}^{\prime}\right\rangle \frac{\partial\left\langle\bar{u}_{i}\right\rangle}{\partial x_{k}}+\frac{\partial\left\langle p^{\prime} u_{i}^{\prime}\right\rangle}{\partial x_{i}}+\frac{1}{2} \frac{\partial\left\langle u_{k}^{\prime} u_{i}^{\prime} u_{i}^{\prime}\right\rangle}{\partial x_{k}}= \\
\nu\left\langle\frac{\partial^{2} u_{i}^{\prime}}{\partial x_{k} \partial x_{k}} u_{i}^{\prime}\right\rangle-\left\langle\left(\frac{\partial \tau_{i k}}{\partial x_{k}}-\left\langle\frac{\partial \tau_{i k}}{\partial x_{k}}\right\rangle\right) u_{i}^{\prime}\right\rangle=\underbrace{\nu\left\langle\frac{\partial^{2} u_{i}^{\prime}}{\partial x_{k} \partial x_{k}} u_{i}^{\prime}\right\rangle}_{\varepsilon^{\text {non }}}-\left\langle\frac{\partial \tau_{i k}}{\partial x_{k}} u_{i}^{\prime}\right\rangle= \\
\nu \frac{\partial^{2} K}{\partial x_{k} \partial x_{k}}-\underbrace{\nu\left\langle\frac{\partial u_{i}^{\prime}}{\partial x_{k}} \frac{\partial u_{i}^{\prime}}{\partial x_{k}}\right\rangle}_{\varepsilon}-\underbrace{\left\langle\frac{\partial \tau_{i k}}{\partial x_{k}} u_{i}^{\prime}\right\rangle}_{\varepsilon_{S G S}}
\end{gathered}
$$

The first term on the right side of the second line is the non-isotropic (i.e. the true) viscous dissipation, $\varepsilon^{\text {non }}$; this is predominately negative. The first term on the last line is the isotropic viscous diffusion term and the second term, $\varepsilon$, is the viscous dissipation term, which is always positive. The last term, $\varepsilon_{S G S}$, is a source term arising from the SGS stress tensor, which can be positive or negative. When it is positive, forward scattering takes place (i.e. it acts as a dissipation term); when it is negative, back scattering occurs. 
Figure 2 presents SGS dissipation, $\varepsilon_{S G S}$ in Eq. 3, computed from filtered DNS data. The forward scatter, $\varepsilon_{S G S}^{+}$, and back scatter, $\varepsilon_{S G S}^{-}$, are defined as the sum of all instants when $\varepsilon_{S G S}$ is positive and negative, respectively. $\varepsilon_{S G S}^{D}$ is computed with $\tau_{i k}^{D}$, see Eq. 7. As can be seen, the scale-similarity model is slightly dissipative (i.e. $\varepsilon_{S G S}>0$ ), but the magnitudes of the forward and the back scatter dissipation are both much larger than $\varepsilon_{S G S}$.

One way to make the SGS stress tensor strictly dissipative is to set the back scatter to zero, i.e. $\max \left(\varepsilon_{S G S}, 0\right)$. This can be achieved by setting $\partial \tau_{i k} / \partial x_{k}=$ 0 when its sign is different from that of $u_{i}^{\prime}$ (see the last term in Eq. 3). This would work if we were solving for $K$. Usually we do not, and the equations that we do solve (the filtered Navier-Stokes equations) are not directly affected by the dissipation term, $\varepsilon_{S G S}$.

Instead we have to modify the SGS stress tensor as it appears in the filtered Navier-Stokes equations, Eq. 1. The second derivative on the right side of Eq. 1 is usually called a diffusion term because it acts like a diffusion transport term. However, when analyzing the stability properties of discretized equations to an imposed disturbance, $u^{\prime}$, using Neumann analysis (see, for example, Chapter 8 in [9]), this term is referred to as a dissipation term. In stability analysis the concern is to dampen numerical oscillations; in connection with SGS models, the aim is to dampen turbulent resolved fluctuations. It is shown in Neumann analysis that the viscous diffusion term in the Navier-Stokes equations is always dissipative, i.e. it dampens numerical oscillations. It is interesting to note the difference in tradition between researchers in numerical analysis and researchers in SGS modelling. The former always consider dissipation by investigating the Navier-Stokes equations. When they devise new discretization schemes to add dissipation (see, for example, Jameson's method $[7,10]$ ), they add an additional flux that corresponds to a (numerical) diffusion term. On the other hand, when researchers in SGS modelling want to add dissipation, they consider the equation for turbulent kinetic energy, $K$. Increased dissipation in their thinking means a larger dissipation term in the $K$ equation. The new scale-similarity model $[3,4]$ was developed by using the approach of both researchers in numerical analysis as well researchers in SGS modelling. The latter approach is applied in choosing the model for the SGS stress tensor, i.e. by the using the scale-similarity model. The sign of the gradient of the SGS stress tensor in the filtered Navier-Stokes equations is chosen by making use of numerical analysis: it is determined so that it behaves as a forcing (or dissipative) term by making sure that is has the opposite (or same) sign as the viscous term in the filtered Navier-Stokes equations.

The viscous dissipation in the $K$ equation stems from the viscous diffusion term in the filtered Navier-Stokes equation. The latter term is always dissipative. This is best seen by looking at Eq. 3. The viscous diffusion term in the filtered Navier-Stokes equation for $u_{i}^{\prime}$ appears in the first term on the second line in Eq. 3. To ensure that $\varepsilon_{S G S}>0$, we set $-\partial \tau_{i k} / \partial x_{k}$ to zero when its sign is different from that of the viscous diffusion term (cf. the two terms on the right side of the second line in Eq. 3). This is achieved by defining a sign function 


$$
M_{i k}^{D}=\operatorname{sign}\left(-\frac{\partial \tau_{i k}}{\partial x_{k}} \frac{\partial^{2} u_{i}^{\prime}}{\partial x_{k} \partial x_{k}}\right), \text { no summation on } i, k
$$

where $M_{i k}^{D}= \pm 1$. The problem is that we do not know $u_{i}^{\prime}\left(=\bar{u}_{i}-\left\langle\bar{u}_{i}\right\rangle\right)$ because $\left\langle\bar{u}_{i}\right\rangle$ is not known until the simulations have been carried out. Fortunately, the sign of the second derivative of the resolved velocity fluctuation, $u_{i}^{\prime}$, is in most cases the same as that of the resolved velocity, $\bar{u}_{i}$. The correlation of the signs of the two second derivatives is larger than $95 \%$ in channel flow for $y^{+}>40$ [3]. Hence Eq. 4 can be replaced by

$$
M_{i k}^{D}=\operatorname{sign}\left(-\frac{\partial \tau_{i k}}{\partial x_{k}} \frac{\partial^{2} \bar{u}_{i}}{\partial x_{k} \partial x_{k}}\right), \text { no summation on } i, k
$$

Each component of the divergence of SGS stress tensor in Eq. 1 is then simply multiplied by

$$
\tilde{M}_{i k}^{D}=\max \left(M_{i k}^{D}, 0\right)
$$

so that

$$
\frac{\partial \tau_{i k}^{D}}{\partial x_{k}}=\tilde{M}_{i k}^{D} \frac{\partial \tau_{i k}}{\partial x_{k}}, \text { no summation on } i, k
$$

By using the limiter, $\tilde{M}_{i k}^{D}$, we omit the back scatter caused by the SGS stresses. Figure 2 includes $\varepsilon_{S G S}^{D}$ which is computed with $\tau_{i k}^{D}$.

\subsection{Back scatter model}

A dissipative scale-similarity model was presented above. Via the sign function, only the dissipative part of the subgrid stress term, $-\partial \tau_{i k} / \partial x_{k}$, was included in the momentum equations. The subgrid stress term can also be used as a forcing term. In this case, only the back scatter part is included. This is easily accomplished by replacing Eqs. 5, 6 and 7 by

$$
\begin{aligned}
M_{i k}^{B} & =-\operatorname{sign}\left(-\frac{\partial \tau_{i k}}{\partial x_{k}} \frac{\partial^{2} \bar{u}_{i}}{\partial x_{k} \partial x_{k}}\right), \text { no summation on } i, k \\
\tilde{M}_{i k}^{B} & =\max \left(M_{i k}^{B}, 0\right) \\
\frac{\partial \tau_{i k}^{B}}{\partial x_{k}} & =\tilde{M}_{i k}^{B} \frac{\partial \tau_{i k}}{\partial x_{k}}, \text { no summation on } i, k
\end{aligned}
$$

where superscript $B$ indicates back scatter. In this way, the subgrid tensor term is included whenever the sign of $-\partial \tau_{i k} / \partial x_{k}$ is opposite to that of the viscous diffusion. This means that the subgrid stress term, $-\partial \tau_{i k}^{B} / \partial x_{k}$, in the momentum equations acts as a counter-gradient diffusion term.

Note that the components of $\partial \tau_{i k}^{B} / \partial x_{k}$ must be added as a volume source (i.e. we cannot integrate the term using the Gauss divergence theorem) because it may be zero and non-zero, respectively, in two adjacent cells. As a consequence, the back-scatter term is not conservative. 


\section{Equations}

\subsection{The turbulence model}

A one-equation model is employed, which reads

$$
\begin{gathered}
\frac{\partial k_{s g s}}{\partial t}+\frac{\partial}{\partial x_{j}}\left(\bar{u}_{j} k_{s g s}\right)=\frac{\partial}{\partial x_{j}}\left[\left(\nu+\nu_{s g s}\right) \frac{\partial k_{s g s}}{\partial x_{j}}\right]+P_{k_{s g s}}-C_{\varepsilon} \frac{k_{s g s}^{3 / 2}}{\Delta} \\
P_{k_{s g s}}=-2 \nu_{s g s} \bar{s}_{i j} \bar{s}_{i j}, \nu_{s g s}=C_{k} k^{1 / 2} \Delta, \Delta=(\Delta V)^{1 / 3}, C_{k}=0.07, C_{\varepsilon}=1.05
\end{gathered}
$$

\subsection{Wall functions}

The wall shear stress is implemented by prescribing the turbulent viscosity at the wall. This is a convenient way of implementing the wall shear stress in general curvi-linear grids. The wall shear stress is defined as

$$
\tau_{w}=\mu \frac{\partial \bar{u}}{\partial y}=\mu_{t, w} \frac{\bar{u}_{P}}{y_{P}}
$$

where $y_{P}$ and $\bar{u}_{P}$ denotes the distance from the wall to the wall-adjacent cell center and the wall-parallel velocity, respectively. Using the definition of the friction velocity, $\tau_{w}=\rho u_{\tau}^{2}$, we obtain

$$
\mu_{t, w} \frac{\bar{u}_{P}}{y_{P}}=\rho u_{\tau}^{2} \rightarrow \mu_{t, w}=\frac{u_{\tau}}{\bar{u}_{P}} \rho u_{\tau} y_{P}
$$

Substituting $u_{\tau} / \bar{u}_{P}$ with the log-law, $\bar{u}_{P} / u_{\tau}=\ln \left(E y^{+}\right) / \kappa$, we can finally write

$$
\mu_{t, w}=\frac{\kappa \rho u_{\tau} y_{P}}{\ln \left(E y^{+}\right)}
$$

We also need a boundary condition for $k_{\text {sgs }}$. In the log-law region, Eq. 9 reduces to the production and the dissipation term, i.e.

$$
\rho C_{\varepsilon} \frac{k_{s g s}^{3 / 2}}{\Delta}=\rho P_{k_{s g s}}=\mu_{s g s}\left(\frac{\partial \bar{u}}{\partial y}\right)^{2}
$$

Multiplying both sides by $\mu_{\text {sgs }}$ gives

$$
\left(\mu_{s g s} \frac{\partial \bar{u}}{\partial y}\right)^{2}=\rho^{2} C_{\varepsilon} \frac{k_{s g s}^{3 / 2}}{\Delta} C_{k} k_{s g s}^{1 / 2} \Delta
$$

The left side is equal to the shear stress up to the power of two, which in the log-law region can be approximated as $\tau_{w}^{2}=\rho^{2} u_{\tau}^{4}$, which gives

$$
k_{s g s, P}=\left(C_{k} C_{\varepsilon}\right)^{-0.5} u_{\tau}^{2}
$$

The wall-adjacent cells should be located in the logarithmic region, i.e. with $y^{+}$values between approximately 30 and 200; the upper limit increases with increasing Reynolds number. 


\begin{tabular}{|c|c|c|c|c|c|c|c|c|}
\hline$R e_{\tau}$ & 4000 & $4000 F$ & 8000 & $8000 F$ & 16000 & $16000 F$ & 32000 & $32000 F$ \\
\hline$\left\langle\tau_{w}\right\rangle$ & 1 & 1.16 & 1 & 1.18 & 1 & 1.18 & 1 & 1.21 \\
$\tau_{w, r m s}^{\prime}$ & 0.23 & 0.49 & 0.25 & 0.51 & 0.19 & 0.5 & 0.19 & 0.54 \\
\hline
\end{tabular}

Table 1. Time averaged and RMS wall shear stress. $F$ denotes forcing.

\subsection{The momentum equations}

The incompressible momentum equation with an added SGS viscosity reads

$$
\frac{\partial \bar{u}_{i}}{\partial t}+\frac{\partial}{\partial x_{j}}\left(\bar{u}_{i} \bar{u}_{j}\right)=-\frac{1}{\rho} \frac{\partial \bar{p}}{\partial x_{i}}+\frac{\partial}{\partial x_{j}}\left(\left(\nu+\nu_{s g s}\right) \frac{\partial \bar{u}_{i}}{\partial x_{j}}\right)-\frac{\partial \tau_{i j}^{B}}{\partial x_{j}}
$$

where the last term is the forcing term, which is non-zero only in the walladjacent cells; $\partial \tau_{i j}^{B} / \partial x_{j}$ is obtained from Eqs. 2 and 8. The forcing term includes the wall-normal derivate, $\partial \tau_{i j}^{B} / \partial x_{2}$. Hence we need to prescribe a numerical wall boundary condition for $\tau_{i j}^{B}$. The second derivative of $\tau_{i j}^{B}$ is set to zero at the wall. For $\tau_{12}^{B}$, for example, this is accomplished by setting the first derivative at the wall equal to that at the wall-adjacent node. For an equidistant grid this gives

$$
\left.\tau_{12}^{B}\right|_{j=1}=\left.1.5 \tau_{12}^{B}\right|_{j=2}-\left.\tau_{12}^{B}\right|_{j=3}
$$

where $j=1$ and $j=2$ denote the wall node and the wall-adjacent node, respectively.

\section{Results}

\subsection{Channel flow}

Fully developed channel flow was computed for four different Reynolds numbers, $R e_{\tau}=u_{\tau} \delta / \nu(\delta$ denotes the half width of the channel $)$, namely $R e_{\tau}=$

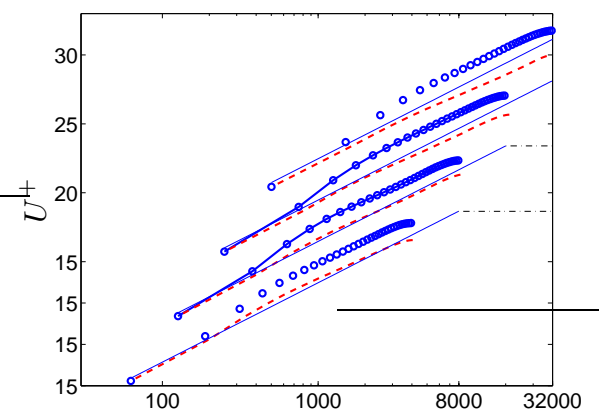

a) $y^{+}$

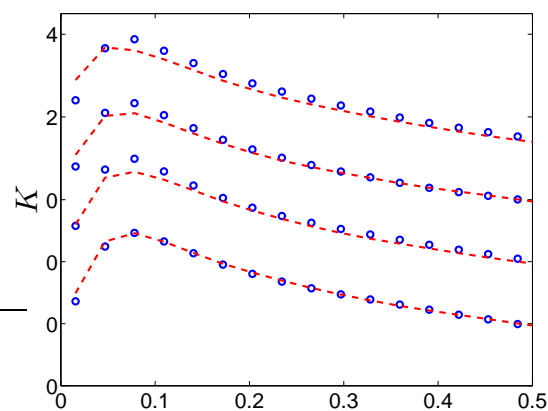

b) $y$

Fig. 3. Channel flow. Mean velocity and resolved turbulent kinetic energy, $K=$ $0.5\left\langle u_{i}^{\prime} u_{i}^{\prime}\right\rangle ;-U^{+}=\left(\ln y^{+}\right) / 0.4+5.2 ;--$ - : forcing; ○: no forcing. From bottom to top in each figure: $R e_{\tau}=4000,8000,16000,32000$. 


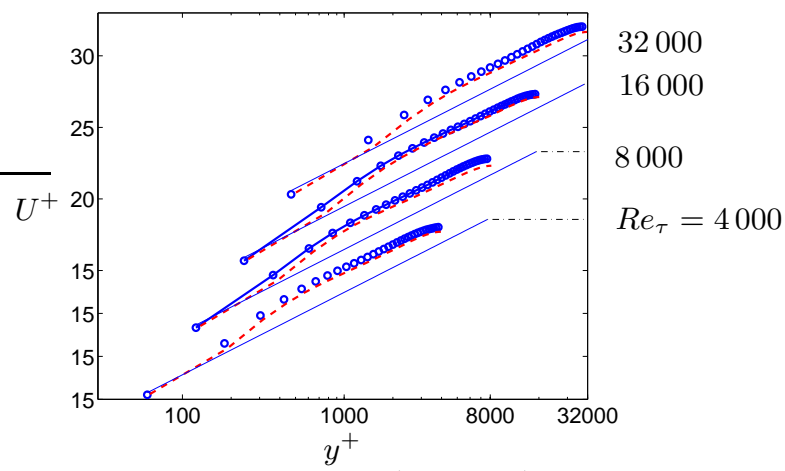

Fig. 4. Channel flow. Mean velocity. $\_: U^{+}=\left(\ln y^{+}\right) / 0.4+5.2 ;---$ : dissipative scale-similarity model, i.e. $\partial \tau_{i j}^{D} / \partial x_{j}$; o: standard scale-similarity model, i.e. $\tilde{M}_{i k}^{D} \equiv 1$.

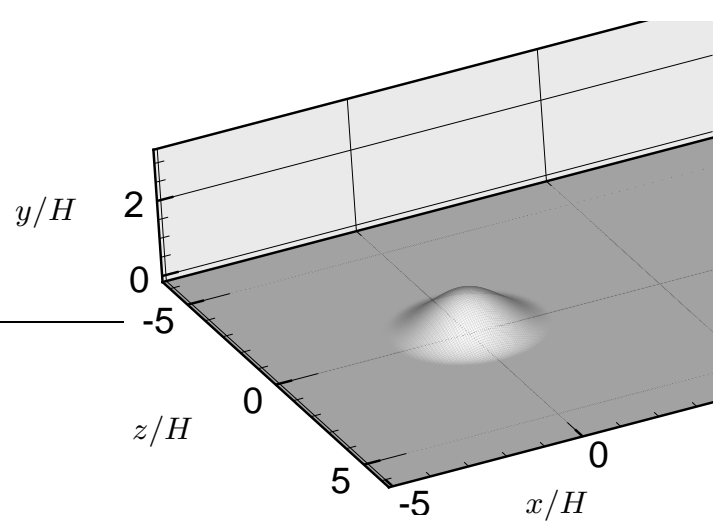

Fig. 5. 3D hill. Computational domain. Hill crest at $x=z=0$. Extension of hill: $-2 \leq x / H \leq 2 ;-2 \leq z / H \leq 2$. Inlet and outlet located at $x / H=-4.1$ and $x / H=15.4$, respectively.

$4000,8000,16000$ and 32000 . The computational extent is $x_{\max }=3.2 \delta$, $y_{\max }=2 \delta$, and $z_{\max }=1.6 \delta$. Constant grid spacing is used in all coordinate directions. The results do not change when the extent and the number of nodes are doubled in $x$ and $z$ directions. Periodic boundary conditions are applied in the streamwise and spanwise directions.

Figure 3a presents the velocity profiles. As can be seen, $U^{+}$decreases when forcing is used, mainly because the wall shear stress increases. Table 1 presents the wall shear stresses and their fluctuations, $\tau_{w, r m s}^{\prime}=\left\langle\tau_{w}^{2}-\left\langle\tau_{w}\right\rangle^{2}\right\rangle^{1 / 2}$. It shows that the wall shear stress increases by approximately $20 \%$ when forcing is applied and that the resolved fluctuations of the wall shear stress increase by $100 \%$ or more.

The resolved turbulent kinetic energy presented in Fig. 3b exhibits a slight decrease when forcing is applied. Forcing usually generates additional resolved turbulence. However, since the forcing is applied only in the wall-adjacent cells, it increases only the wall shear stress and generates additional fluctuations in the wall shear stress. 

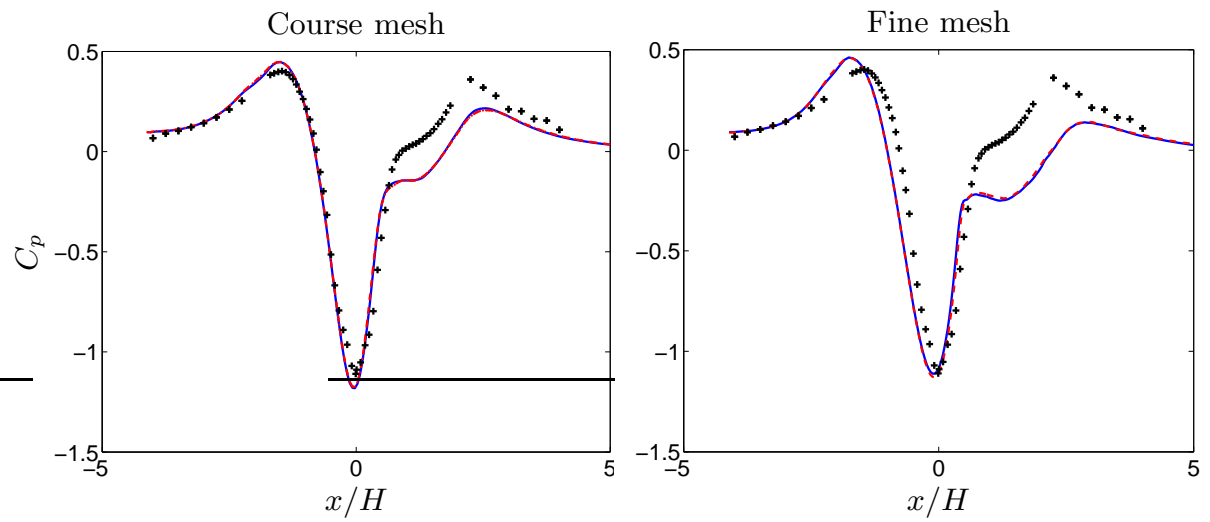

Fig. 6. 3D hill flow. Pressure coefficient.

: no forcing; - - - : forcing; ; +: experiments [13]

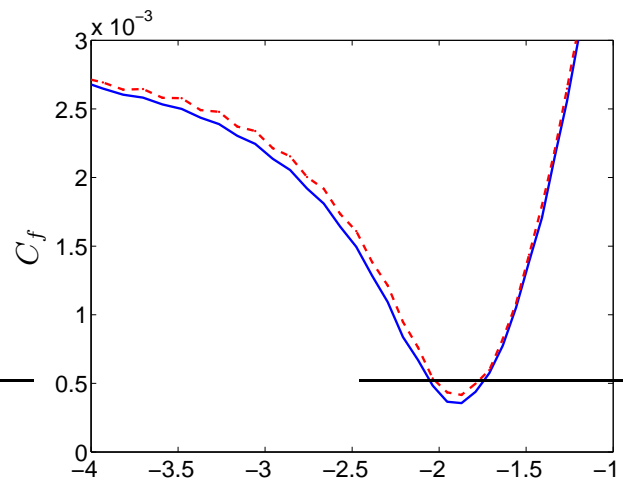

a) $x / H$

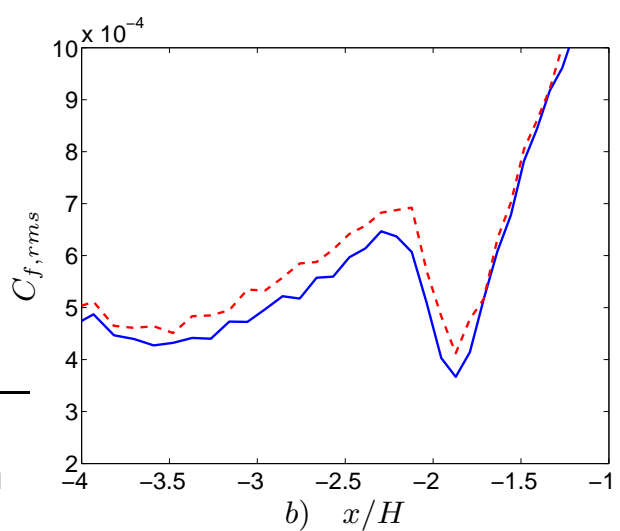

Fig. 7. 3D hill flow. Wall friction coefficient (a) and its root-mean-square (b). Coarse mesh. Zoom in front of the hill. : no forcing; - - - : forcing.

\section{$5.23 \mathrm{D}$ hill flow}

The flow around the 3D hill was also computed using wall functions. The Reynolds number is 130000 based on the hill height. The coarse mesh has $160 \times 80 \times 130$ cells (streamwise, $x$; wall-normal, $y$; spanwise, $z$ ). The fine mesh has $210 \times 80 \times 210$ cells (it is refined in the region $0<x / H<2$, $-2<z / H<2$ ). The wall-adjacent cells are located so that $30<y^{+}<60$. A weak stretching of approximately $3 \%$ is used in the wall-normal direction, which gives $(\Delta x / \Delta y)_{\max }<6$ and $(\Delta x / \Delta y)_{\max }<4$ in the hill region $(|x|<4$, $|z|<2)$ for both meshes. Forcing cannot be applied over the entire low-wall region because this causes divergence. Instead, forcing is applied only outside the hill region, i.e. $|x|<2$ and $|z|<2$. Synthetic fluctuations are used at the inlet for creating inlet turbulence [2].

The pressure coefficient for both meshes is presented in Fig. 6. As can be seen, the effect of forcing is negligible for both meshes. The agreement with experiments is poor; it should be mentioned that good agreement with 
experiments using wall functions has been shown in the literature [16]. These authors used a different SGS model (a dynamic Smagorinsky) but the mesh $(192 \times 96 \times 192$ cells $)$ was fairly similar to the present fine mesh.

Figure 7 presents the wall friction coefficient and its RMS in front of the hill. As expected, forcing does give a small increase in $C_{f}$ and a slightly larger $C_{f, r m s}$.

\section{Concluding Comments}

LES with wall functions have been presented. A new approach improving wall functions has been proposed: forcing is applied at the cells adjacent to the walls. The method was validated for fully developed channel flow and 3D hill flow. For channel flow, forcing increases the resolved fluctuations (RMS) of the wall shear stress by more than a factor of two, and the resolved wall shear stresses are increased by some $20 \%$.

However, for the 3D hill flow, the effect of forcing is unfortunately negligible. There is a small increase in the wall friction coefficient and its resolved fluctuations, but there is no effect on the mean flow. One reason may be that the separation process to a large degree is governed by geometry. This flow is indeed an extremely challenging flow [8] and maybe it should not be expected that wall-functions should be able to handle this flow. It is expected that the new wall functions work better in flows where the separation process is governed by the delicate balance between momentum diffusion and pressure gradient. However, the author is not aware of any such test cases at high Reynolds number.

\section{References}

1. Batten, P., Goldberg, U., Chakravarthy, S.: Interfacing statistical turbulence closures with large-eddy simulation. AIAA Journal 42(3), 485-492 (2004)

2. Davidson, L.: Using isotropic synthetic fluctuations as inlet boundary conditions for unsteady simulations. Advances and Applications in Fluid Mechanics 1(1), 1-35 (2007)

3. Davidson, L.: Hybrid LES-RANS: back scatter from a scale-similarity model used as forcing. Phil. Trans. of the Royal Society A 367(1899), 2905-2915 (2009)

4. Davidson, L.: A dissipative scale-similarity model. In: V. Armenio, B. Geurts, J. Fröhlich (eds.) DLES7: Direct and Large-Eddy Simulations 7, ERCOFTAC series, vol. 13, pp. 261-266 (2010)

5. Davidson, L., Billson, M.: Hybrid LES/RANS using synthesized turbulence for forcing at the interface. International Journal of Heat and Fluid Flow 27(6), 1028-1042 (2006)

6. Davidson, L., Dahlström, S.: Hybrid LES-RANS: An approach to make LES applicable at high Reynolds number. International Journal of Computational Fluid Dynamics 19(6), 415-427 (2005) 
7. Esfahaniana, V., Akbarzade, P.: The Jameson's numerical method for solving the incompressible viscous and inviscid flows by means of artificial compressibility and preconditioning method. Applied Mathematics and Computation 206(2), 651-661 (2008)

8. Garca-Villalba, M., Rodi, W., Leschziner, M.: Large-eddy simulation of separated flow over a three-dimensional axisymmetric hill. Journal of Fluid Mechanics 627, 55-96 (2009)

9. Hirsch, C.: Numerical Computation of Internal and External Flows: Fundamentals of Numerical Discretization, vol. 1. John Wiley \& Sons, Chichester, UK (1988)

10. Jameson, A.: Steady-state solution of the Euler equations for transonic flow. Advances in Scientific Computing pp. 37-69 (1982)

11. Keating, A., Piomelli, U.: A dynamic stochastic forcing method as a wall-layer model for large-eddy simulation. Journal of Turbulence 7(12), 1-25 (2008)

12. Piomelli, U., Balaras, E., Pasinato, H., Squire, K., Spalart, P.: The inner-outer layer interface in large-eddy simulations with wall-layer models. International Journal of Heat and Fluid Flow 24, 538-550 (2003)

13. Simpson, R., Long, C.H., Byun, G.: Study of vortical separation from an axisymmetric hill. International Journal of Heat and Fluid Flow 23(5), 582-591 (2002)

14. Speziale, C.: Galilean invariance of subgrid-scale stress models in the large-eddy simulation of turbulence. Journal of Fluid Mechanics 156, 55-62 (1985)

15. Temmerman, L., Hadiabdi, M., Leschziner, M., Hanjalic, K.: A hybrid two-layer URANS-LES approach for large eddy simulation at high Reynolds numbers. International Journal of Heat and Fluid Flow 26(2), 173-190 (2005)

16. Tessicini, F., Li, N., Leschziner, M.: Large-eddy simulation of three-dimensional flow around a hill-shaped obstruction with a zonal near-wall approximation. International Journal of Heat and Fluid Flow 28(5), 894-908 (2007) 